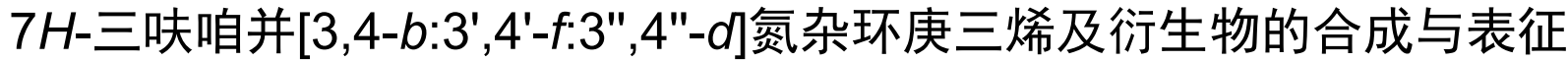

$\begin{array}{ccc}\text { 贾思媛 } & \begin{array}{c}\text { 张海昊 } \\ \text { 李祥志 } \\ \text { (西安近代化学研究所 西安 710065) }\end{array}\end{array}$

\begin{abstract}
摘要 以 3,4-双(3'-氨基呋咱-4'-基)呋咱(BATF)为原料, 经 Caro's acid 氧化制备了 3,4-双(3'-硝基呋咱-4'-基)呋咱(BNTF), 收率为 $93 \%$; 基于 $\mathrm{BNTF}$ 分子中的 $\mathrm{NO}_{2}$ 反应性，与水合肼、氨水等发生亲核取代、环化反应，合成了含能化合物 $7 H$ -

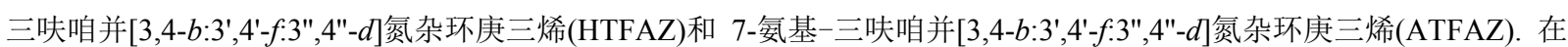
硝酸-醋䣶体系中氧化 HTFAZ 得到新化合物 7,7'二(呋咱[3,4-b:3,4'-d:3",4"-f]氮杂环庚三烯)(BTFZ), 采用红外光谱、核 磁共振、元素分析和质谱等进行了结构表征. 培养了 HTFAZ 单晶, $X$ 射线单晶衍射分析表明: HTFAZ 晶体结构属单斜 晶系, 空间群为 $P 2(1) / n, a=0.7252(15) \mathrm{nm}, b=0.6458(14) \mathrm{nm}, c=1.6814(4) \mathrm{nm}, V=0.7781(3) \mathrm{nm}^{3}, Z=4, D_{\mathrm{c}}=1.871 \mathrm{~g} \cdot$ $\mathrm{cm}^{-3}, F(000)=440, R_{1}=0.0353, w R_{2}=0.0895$. 开展了 HTFAZ, ATFAZ 和 BTFZ 的物化、爆轰性能和热性能研究, 其中 HTFAZ 密度 $1.871 \mathrm{~g} \cdot \mathrm{cm}^{-3}$ 、生成焓 $733.7 \mathrm{~kJ} \cdot \mathrm{mol}^{-1}$ 、熔点 $193.1{ }^{\circ} \mathrm{C}$ 、分解点 $345.1{ }^{\circ} \mathrm{C}$ 、爆速 $8416.5 \mathrm{~m} \cdot \mathrm{s}^{-1}$ 、爆压为 32.9 GPa; ATFAZ 密度 $1.788 \mathrm{~g} \bullet \mathrm{cm}^{-3}$ 、生成焓 $831.2 \mathrm{~kJ} \bullet \mathrm{mol}^{-1}$ 、熔点 $204 \sim 207{ }^{\circ} \mathrm{C}$ 、分解点 $323.7{ }^{\circ} \mathrm{C}$ 、爆速 $8300 \mathrm{~m} \bullet \mathrm{s}^{-1}$ 、爆压 为 $30 \mathrm{GPa}$; BTFZ 密度 $1.862 \mathrm{~g} \cdot \mathrm{cm}^{-3}$ 、生成焓 $1752.4 \mathrm{~kJ} \cdot \mathrm{mol}^{-1}$ 、分解点 $280.2{ }^{\circ} \mathrm{C}$ 、爆速 $7924.5 \mathrm{~m} \cdot \mathrm{s}^{-1}$ 、爆压为 $28.42 \mathrm{GPa}$. 关键词 $7 H$-三呋咱并[3,4-b:3',4'-f:3",4"-d] 氮杂环庚三烯; 衍生物; 合成; 晶体结构; 性能
\end{abstract}

\section{Synthesis and Characterization of $7 \mathrm{H}$-Trifurazano- $\left[3,4-b: 3^{\prime}, 4^{\prime}-f: 3^{\prime \prime}, 4^{\prime \prime}-d\right]$ azepine and Its Analogues}

\author{
Jia, Siyuan Zhang, Haihao Zhou, Cheng Lai, Weipen \\ Li, Xiangzhi Wang, Bozhou* \\ (Xi'an Modern Chemistry Research Instritute, Xi'an 710065)
}

\begin{abstract}
Using 3,4-bis(4'-aminofurazano-3'-yl)furazan (BATF) as the starting material, 3,4-bis(4'-nitrofurazano-3'-yl)- furazan (BNTF) was synthesized by Caro's acid oxidation with a yield of $93 \%$. Based on the reactivity of $\mathrm{NO}_{2}$ in $\mathrm{BNTF}^{2}$, two energetic material 7H-trifurazano[3,4-b:3',4'-f:3",4"-d] azepine (HTFZA) and 7-aminotrifurazano[3,4-b:3',4'-f:3",4"-d]azepine (ATFAZ) were designed and synthesized from BNTF and $\mathrm{NH}_{3} \cdot \mathrm{H}_{2} \mathrm{O}$ or $\mathrm{NH}_{2} \mathrm{NH}_{2} \cdot \mathrm{H}_{2} \mathrm{O}$ via the nucleophilic substitution and cyclization, then HTFAZ was nitrated in the system of $\mathrm{HNO}_{3}-\mathrm{Ac}_{2} \mathrm{O}$ to firstly obtain a novel compound 7,7'-bis(terfurazan $\left[3,4-b: 3,4^{\prime}-d: 3 ", 4 "-f\right]$ azepine) (BTFZ). The above three compounds were characterized by the means of IR, ${ }^{1} \mathrm{H}$ NMR, ${ }^{13} \mathrm{C}$ NMR, MS and elemental analysis. The single crystal of HTFAZ was studied by X-ray single crystal diffraction. Its crystal belongs to orthorhombic, space group is $P 2(1) / n$ with $a=0.7252(15) \mathrm{nm}, b=0.6458(14) \mathrm{nm}, c=1.6814(4) \mathrm{nm}, V=0.7781(3) \mathrm{nm}^{3}, Z=4$, $D_{\mathrm{c}}=1.871 \mathrm{~g} \bullet \mathrm{cm}^{-3}, F(000)=440, R_{1}=0.0353$ and $w R_{2}=0.0895$. The properties of HTFAZ, ATFAZ and BTFZ were measured

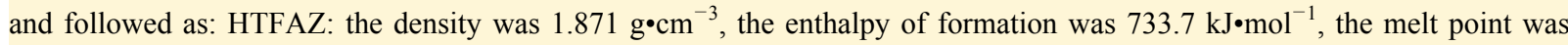
193.1 ${ }^{\circ} \mathrm{C}$, the decomposition point was $345.1{ }^{\circ} \mathrm{C}$, the explosion velocity was $8416.5 \mathrm{~m} \cdot \mathrm{s}^{-1}$, the detonation pressure was 32.9

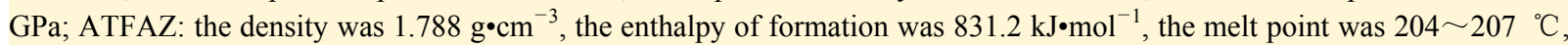
the decomposition point was $323.7^{\circ} \mathrm{C}$, the explosion velocity was $8300 \mathrm{~m} \bullet \mathrm{s}^{-1}$, the detonation pressure was $30 \mathrm{GPa}$; BTFZ: the density was $1.862 \mathrm{~g} \cdot \mathrm{cm}^{-3}$, the enthalpy of formation was $1752.4 \mathrm{~kJ} \cdot \mathrm{mol}^{-1}$, the decomposition point was $280.2{ }^{\circ} \mathrm{C}$, the explosion velocity was $7924.5 \mathrm{~m} \cdot \mathrm{s}^{-1}$, the detonation pressure was $28.42 \mathrm{GPa}$.
\end{abstract}

Keywords 7-aminotrifurazano[3,4- $\left.b: 3^{\prime}, 4^{\prime}-f: 3^{\prime \prime}, 4^{\prime \prime}-d\right]$ azepine; analogues; synthesis; crystal structure; property

\footnotetext{
* E-mail: wbz600@163.com

Received September 5, 2014; revised October 11, 2014; published online December 15, 2014.

Project supported by the National Natural Science Foundation of China (No. 21243007).

国家自然科学基金(No. 21243007)资助项目.
} 
呋咱环是构建新型高能量密度材料的理想结构单 元，基于此结构单元可合成多种性能优异的呋咱化合 物. 含能呋咱化合物一般具有能量高、密度大、标准生 成焓高、氮含量高、氧平衡好等特点 ${ }^{[1 \sim 7]}$. 3,4-双( $3^{\prime}$-硝基 呋咱-4'-基)氧化呋咱(DNTF)为性能优异的新型呋咱含 能化合物 ${ }^{[8]}$, 通过理论分析发现, 在 DNTF 结构单元中, 由于氧化呋咱环的配位氧化学性质较为活泼, 从而导致 DNTF 冲击波感度偏高. 本课题组 ${ }^{[9]}$ 于 2011 年成功合成 了 DNTF 去配位氧化合物 3,4-双(3'-硝基呋咱-4'-基)呋咱 (BNTF), 性能测试结果表明, BNTF 能量水平略低于 DNTF, 冲击波感度较 DNTF 明显降低, 熔点较低可以 用于熔铸炸药的液相载体. 同时, 利用 DNTF 硝基的反 应活性, 设计、合成了一系列环状化合物如三呋咱并氧 杂环庚三烯, 如双呋咱并 $\left[3,4-b: 3^{\prime}, 4-f\right]$ 氧化呋咱并 $\left[3^{\prime \prime,} 4^{\prime \prime}-\right.$ $d]$ 氧杂环庚三烯、7-氨基-三呋咱 $\left[3,4-b: 3,4^{\prime}-d: 3^{\prime \prime}, 44^{\prime \prime}-f\right.$ 氮杂 环庚三烯-1-氧化物和 $7 H$-三呋咱 $\left[3,4-b: 3,4^{\prime}-d: 3^{\prime \prime}, 4 "-f\right]$ 氮 杂环庚三烯 1-氧化物等 ${ }^{[10 \sim 12]}$.

本工作参照文献 $[13,14]$, 利用 BNTF 结构中硝基的 反应活性, 合成了新型含能材料 $7 H$-三呋咱并 $\left[3,4-b: 3^{\prime}, 4^{\prime}-f: 3^{\prime \prime}, 4^{\prime \prime}-d\right]$ 氮杂环庚三烯(HTFAZ)和 7-氨基-三 呋咱并 $\left[3,4-b: 3^{\prime}, 4^{\prime}-f: 3^{\prime \prime}, 4^{\prime \prime}-d\right]$ 氮杂环庚三烯 (ATFAZ). 以 HTFAZ 为原料经 $\mathrm{HNO}_{3}-\mathrm{Ac}_{2} \mathrm{O}$ 体系氧化得到了未见文献 报道的新化合物 7,7'-二(呋咱[3,4- $\left.b: 3,4^{\prime}-d: 3 ", 4^{\prime \prime}-f\right]$ 氮杂环 庚三烯)(BTFZ) (Scheme 1), 采用红外、核磁、元素和质 谱等对目标化合物及其中间体进行了结构表征. 培养了
HTFAZ 单晶，证实了 HTFAZ 具有七元氮杂环结构. 探 讨了 BNTF 环化反应和 HTFAZ 氧化反应机理, 并系统 研究了反应温度、时间等关键因素对 HTFAZ 收率的影 响, 确定了适宜的反应条件. 通过理论计算获得了 HTFAZ, ATFAZ 和 BTFZ 的部分物化与爆轰性能, 采用 差示扫描量热法(DSC)和热失重(TGA)研究了ATFAZ和 BTFZ 的热行为, 为进一步开展应用研究提供必要的基 础数据.

\section{1 结果与讨论}

\section{1 反应机理探讨}

\subsection{1 分子内环化反应机理}

由于 $\mathrm{BNTF}$ 分子中 $\mathrm{NO}_{2}$ 强吸电子作用以及呋咱环的 诱导作用，导致与硝基相连的碳原子电子云密度降低， 显示出部分正电性，有利于亲核试剂的进攻. $\mathrm{NH}_{3}$ 具有 一对孤对电子，极易进攻 $\mathrm{C}-\mathrm{NO}_{2}$ 中显正电性的 $\mathrm{C}$ 原子, 同时失去 1 分子 $\mathrm{HNO}_{2}$, 而形成氨基; 同时，新形成的 $\mathrm{NH}_{2}$ 同样具有一对孤对电子，可进攻另外一个 $\mathrm{C}-\mathrm{NO}_{2}$ 中显正电性的 $\mathrm{C}$ 原子, 失去 1 分子 $\mathrm{HNO}_{2}$, 环化生成目 标产物 HTFAZ, 由于 BNTF 的晶体结构类似于 DNTF, 晶体结构中分子呈环状 ${ }^{[15,16]}$, 其中两个硝基比较靠近, 并非直链状，因此发生亲核取代反应时亲核试剂容易和 较近的两个硝基环化，而非形成直链状化合物. 推测其 反应机理如 Scheme 2 .

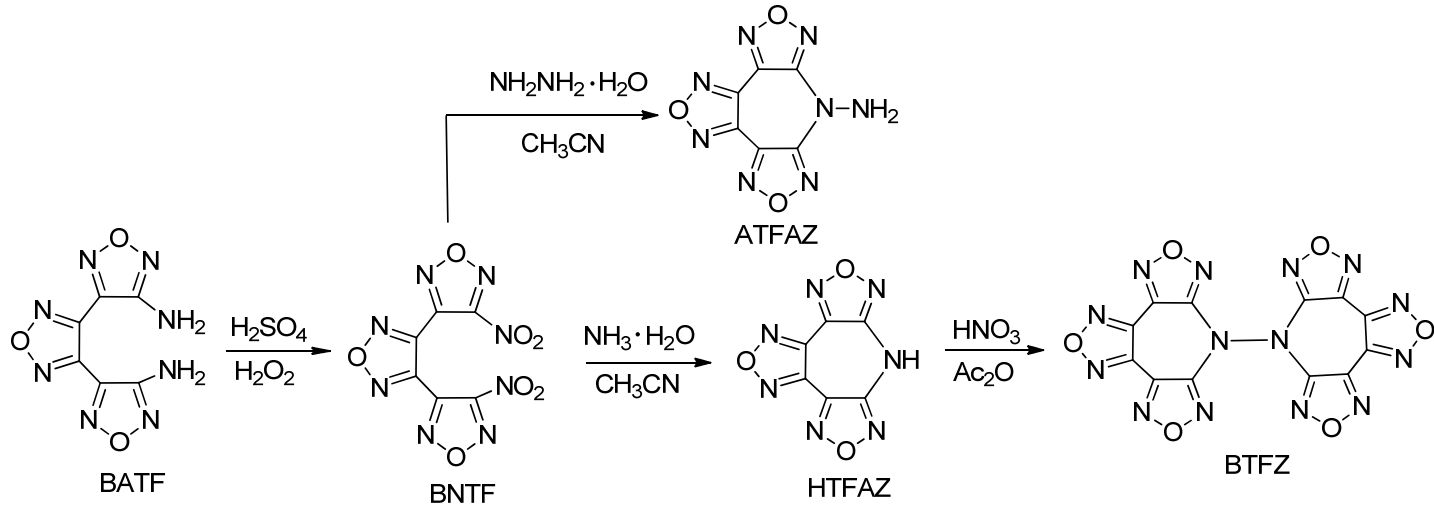

图式 1 化合物的合成路线

Scheme 1 Synthetic route of compounds
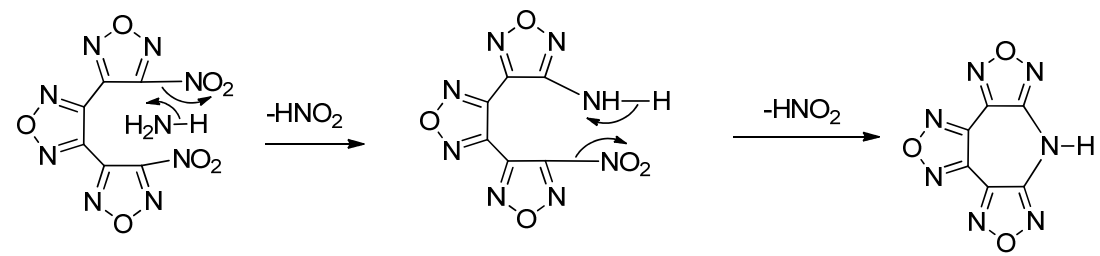

图式 2 HTFAZ 分子内成环反应机理

Scheme 2 Intramolecular cyclization mechanism of HTFAZ 


\subsubsection{HTFAZ 氧化反应机理}

醋酎与硝酸反应生成醋酸硝酸酯, 然后进一步生成 硝酰阳离子 $\left(\mathrm{NO}_{2}^{+}\right), \mathrm{NO}_{2}^{+}$与 HTFAZ 中的亚胺 $\mathrm{N}$ 原子, 生成过渡态 1 , 接着脱去 $\mathrm{H}^{+}$生成 7-硝基-三呋咱并[3,4$\left.b: 3^{\prime}, 4^{\prime}-f: 3^{\prime}, 4^{\prime \prime}-d\right]$ 氮杂环庚三烯(2). 由于硝基化合物 2 极 不稳定, HTFAZ 中亚胺 $\mathrm{N}$ 原子上的孤对电子进攻硝基 化合物 2 显正电性的 $\mathrm{N}$ 原子, 同时脱去 1 分子 $\mathrm{HNO}_{2}$ 得 到目标化合物 BTFZ. 推测其反应机理如 Scheme 3.
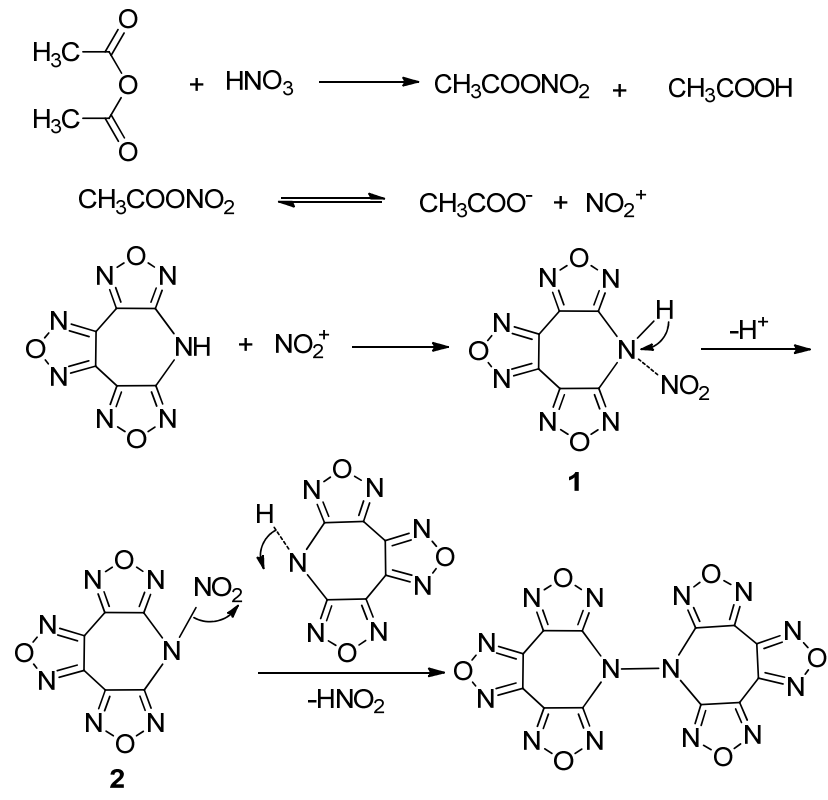

图式 3 HTFAZ 氧化反应机理

Scheme 3 Oxidation mechanism of HTFAZ

\section{2 亲核取代反应条探讨}

亲核取代反应中亲核试剂提供一对电子与底物碳 原子成键, 亲核试剂给电子能力强, 成键快, 即亲核性 强. 亲核性强弱是影响反应收率的重要因素. 一般来说, 试剂的碱性强, 亲和能力也强. 很多情况下碱性与亲核 性是一致的 ${ }^{[17]}$. 在相同的溶剂中水合肼的碱性较强, 氨 水的碱性相对较弱, 水合肼的亲核性更强, 因此反应过 程中, BNTF 与水合肼反应产物收率较高达到 $71 \%$, 与 氨水反应收率较低.

\section{3 反应条件优化}

\subsection{1 反应温度的影响}

在反应时间为 $2 \mathrm{~h}$ 条件下, 研究了不同反应温度对 HTFAZ 收率的影响, 具体结果见表 1.

由表 1 可以看出, 当反应温度为 $25 \sim 30{ }^{\circ} \mathrm{C}$ 时, 收 率最高达到 54\%; 继续提高反应温度, 收率反而降低, 通过薄层色谱分析跟踪实验发现提高温度可能导致副 反应增加, 副产物增多导致收率下降, 因此适宜反应温 度应为 $25 \sim 30{ }^{\circ} \mathrm{C}$.
表 1 反应温度对 HTFAZ 收率的影响

Table 1 Effect of reaction temperature on yield of HTFAZ

\begin{tabular}{cc}
\hline Temperature $/{ }^{\circ} \mathrm{C}$ & Yield $/ \%$ \\
\hline 20 & 48 \\
25 & 54 \\
30 & 54 \\
40 & 52 \\
50 & 50 \\
\hline
\end{tabular}

1.3.2 反应时间的影响

在反应温度为 $25 \sim 30{ }^{\circ} \mathrm{C}$ 条件下, 研究了不同反应 时间对 HTFAZ 收率的影响, 具体结果见表 2.

表 2 反应时间对 HTFAZ 收率的影响

Table 2 Effect of reaction time on yield of HTFAZ

\begin{tabular}{cc}
\hline Time $/ \mathrm{h}$ & Yield/\% \\
\hline 1 & 48 \\
2 & 54 \\
3 & 52 \\
4 & 50 \\
\hline
\end{tabular}

从表 2 中可以看出, 当反应时间 $1 \mathrm{~h}$ 时，反应收率为 $48 \%$ ，薄层色谱分析发现原料反应不完全; 当反应时间 为 $2 \mathrm{~h}$, 收率可达到最高 $(54 \%)$; 进一步继续延长反应时 间, 实验发现收率降低, 究其原因可能是 BNTF 在碱性 条件下会分解成双肜化合物.

\subsection{HTFAZ 的晶体结构分析}

HTFAZ 的晶体参数、数据收集及结构修正列于表 3 , 分子结构及其在晶胞中的堆积方式见图 1, 部分键 长、键角及二面角、氢键见表 $4 \sim 6$.

表 3 HTFAZ 的晶体结构数据

Table 3 Crystal structure data of HTFAZ

\begin{tabular}{ll}
\hline Formula & $\mathrm{C}_{6} \mathrm{HN}_{7} \mathrm{O}_{3}$ \\
Formula weight & 219.14 \\
Crystal system & Monoclinic \\
Space group & $P 2(1) / n$ \\
$a / \mathrm{nm}$ & $0.7252(15)$ \\
$b / \mathrm{nm}$ & $0.6458(14)$ \\
$c / \mathrm{nm}$ & $1.6814(4)$ \\
$V / \mathrm{nm}^{3}$ & $0.7781(3)$ \\
$Z$ & 4 \\
$D_{\text {calcd }} /\left(\mathrm{g} \bullet \mathrm{cm}^{-3}\right)$ & 1.871 \\
$F(000)$ & 440 \\
$\lambda / \mathrm{nm}$ & 0.71073 \\
Completeness to $\theta=28.14^{\circ}$ & $97.2 \%$ \\
Reflections collected/unique & $4607 / 1875$ \\
Goodness-of-fit on $F^{2}$ & 1.046 \\
Final $R$ indexes $[I>2 \sigma(I)]$ & 0.0353 \\
Final $R$ indexes (all data) & 0.0502 \\
Largest diff peak and hole/(e•nm $\left.{ }^{-3}\right)$ & 0.257 and -0.145 \\
\hline
\end{tabular}

从图 1 可以看出, HTFAZ 分子结构中三个呋咱环经 
(a)
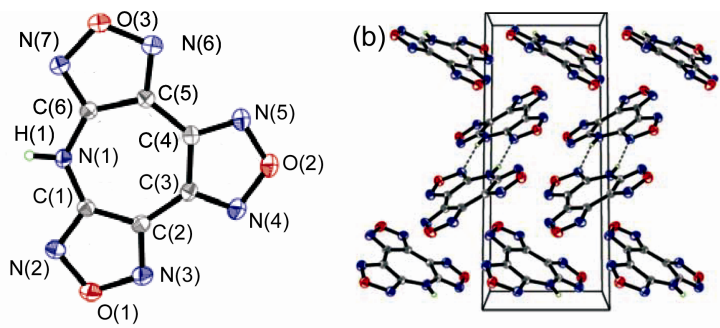

图 1 HTFAZ 的分子结构(a)和晶胞堆积图(b)

Figure 1 Moleculer structure (a) and packing view (b) of HTFAZ

表 4 HTFAZ 的部分键长与键角

Table 4 Selected bond lengths (nm) and bond angles $\left({ }^{\circ}\right)$ of HTFAZ

\begin{tabular}{lc||ll}
\hline $\mathrm{C}(1)-\mathrm{N}(2)$ & $0.1303(18)$ & $\mathrm{N}(2)-\mathrm{C}(1)-\mathrm{N}(1)$ & $119.57(13)$ \\
$\mathrm{C}(1)-\mathrm{N}(1)$ & $0.1370(18)$ & $\mathrm{N}(2)-\mathrm{C}(1)-\mathrm{C}(2)$ & $109.39(12)$ \\
$\mathrm{C}(1)-\mathrm{C}(2)$ & $0.1426(2)$ & $\mathrm{N}(1)-\mathrm{C}(1)-\mathrm{C}(2)$ & $131.03(13)$ \\
$\mathrm{N}(2)-\mathrm{O}(1)$ & $0.1391(16)$ & $\mathrm{N}(3)-\mathrm{C}(2)-\mathrm{C}(1)$ & $108.68(13)$ \\
$\mathrm{N}(3)-\mathrm{O}(1)$ & $0.1367(17)$ & $\mathrm{N}(3)-\mathrm{C}(2)-\mathrm{C}(3)$ & $123.08(13)$ \\
$\mathrm{C}(2)-\mathrm{N}(3)$ & $0.1303(18)$ & $\mathrm{C}(1)-\mathrm{C}(2)-\mathrm{C}(3)$ & $128.23(13)$ \\
$\mathrm{C}(2)-\mathrm{C}(3)$ & $0.1458(2)$ & $\mathrm{N}(4)-\mathrm{C}(3)-\mathrm{C}(4)$ & $109.36(13)$ \\
$\mathrm{C}(3)-\mathrm{N}(4)$ & $0.1306(18)$ & $\mathrm{N}(4)-\mathrm{C}(3)-\mathrm{C}(2)$ & $124.02(13)$ \\
$\mathrm{C}(3)-\mathrm{C}(4)$ & $0.1413(19)$ & $\mathrm{C}(4)-\mathrm{C}(3)-\mathrm{C}(2)$ & $126.61(12)$ \\
$\mathrm{C}(4)-\mathrm{N}(5)$ & $0.1305(18)$ & $\mathrm{N}(5)-\mathrm{C}(4)-\mathrm{C}(3)$ & $109.04(13)$ \\
$\mathrm{C}(4)-\mathrm{C}(5)$ & $0.1455(2)$ & $\mathrm{N}(5)-\mathrm{C}(4)-\mathrm{C}(5)$ & $123.66(13)$ \\
\hline
\end{tabular}

表 5 HTFAZ 的部分二面角

Table 5 Selected torsion angles $\left({ }^{\circ}\right)$ of HTFAZ

\begin{tabular}{ll}
$\mathrm{N}(2)-\mathrm{C}(1)-\mathrm{C}(2)-\mathrm{N}(3)$ & $0.59(16)$ \\
$\mathrm{N}(1)-\mathrm{C}(1)-\mathrm{C}(2)-\mathrm{N}(3)$ & $-179.85(15)$ \\
$\mathrm{C}(2)-\mathrm{C}(1)-\mathrm{N}(2)-\mathrm{O}(1)$ & $-0.87(15)$ \\
$\mathrm{C}(1)-\mathrm{C}(2)-\mathrm{N}(3)-\mathrm{O}(1)$ & $-0.01(15)$ \\
$\mathrm{C}(3)-\mathrm{C}(2)-\mathrm{N}(3)-\mathrm{O}(1)$ & $179.33(12)$ \\
$\mathrm{N}(6)-\mathrm{C}(5)-\mathrm{C}(6)-\mathrm{N}(1)$ & $179.84(14)$ \\
$\mathrm{C}(1)-\mathrm{C}(2)-\mathrm{C}(3)-\mathrm{C}(4)$ & $3.1(2)$ \\
$\mathrm{C}(2)-\mathrm{C}(3)-\mathrm{C}(4)-\mathrm{C}(5)$ & $3.4(2)$ \\
$\mathrm{C}(3)-\mathrm{C}(4)-\mathrm{C}(5)-\mathrm{C}(6)$ & $-6.8(2)$ \\
$\mathrm{N}(1)-\mathrm{C}(6)-\mathrm{N}(7)-\mathrm{O}(3)$ & $-179.49(12)$ \\
$\mathrm{N}(1)-\mathrm{C}(1)-\mathrm{N}(2)-\mathrm{O}(1)$ & $179.51(12)$ \\
$\mathrm{C}(4)-\mathrm{C}(5)-\mathrm{C}(6)-\mathrm{N}(1)$ & $-2.5(2)$ \\
\hline
\end{tabular}

表 6 HTFAZ 的部分氢键的键长 $(\mathrm{nm})$ 和键角 $\left(^{\circ}\right)$

Table 6 Bond lengths (nm) and bond angles $\left({ }^{\circ}\right)$ of hydrogen bonds for HTFAZ

\begin{tabular}{lcccc}
\hline $\mathrm{D}-\mathrm{H} \cdots \mathrm{A}$ & $\mathrm{D}-\mathrm{H}$ & $\mathrm{H} \cdots \mathrm{A}$ & $\mathrm{D} \cdots \mathrm{A}$ & $\mathrm{DHA}$ \\
\hline $\mathrm{N}(1)-\mathrm{H}(1) \cdots \mathrm{N}(7) \# 1$ & $0.084(2)$ & $0.219(2)$ & $3.0065(18)$ & $165.7(19)$ \\
\hline
\end{tabular}

仲氨基连接成一个七元环. 从表 4 可以看出, 组成呋咱 环 $\mathrm{C}-\mathrm{C}, \mathrm{C}=\mathrm{N}$ 和 $\mathrm{N}-\mathrm{O}$ 键的平均键长分别为 0.1426 , 0.1369 和 $0.1367 \mathrm{~nm}$, 与文献报道值基本一致 ${ }^{[18,19]}$, 二面 角 $\mathrm{N}(2)-\mathrm{C}(1)-\mathrm{C}(2)-\mathrm{N}(3), \mathrm{N}(1)-\mathrm{C}(1)-\mathrm{C}(2)-\mathrm{N}(3)$, $\mathrm{C}(1)-\mathrm{C}(2)-\mathrm{N}(3)-\mathrm{O}(1), \mathrm{C}(2)-\mathrm{C}(1)-\mathrm{N}(2)-\mathrm{O}(1)$ 分别
为 $0.59^{\circ},-179.85^{\circ},-0.01^{\circ},-0.87^{\circ}$. 上述数据显示呋 咱环共平面, 呋咱环上 6 个电子形成一个共轭 $\pi$ 键, 其 中每个 $\mathrm{C}$ 原子提供 1 个电子, 每个 $\mathrm{N}$ 原子提供 1 电子, 氧原子上有一对孤对电子.

从表 5 中二面角 $\mathrm{C}(1)-\mathrm{C}(2)-\mathrm{C}(3)-\mathrm{C}(4)\left(3.1^{\circ}\right)$, $\mathrm{C}(2)-\mathrm{C}(3)-\mathrm{C}(4)-\mathrm{C}(5)\left(3.4^{\circ}\right), \mathrm{C}(3)-\mathrm{C}(4)-\mathrm{C}(5)-\mathrm{C}(6)$ $\left(-6.8^{\circ}\right)$ 可以看出三个呋咱环共面. 其实三个呋咱环所形 成的三个平面: 平面 $1[\mathrm{C}(1)-\mathrm{C}(2)-\mathrm{N}(3)-\mathrm{O}(1)-\mathrm{N}(2)]$ 与平面 $2[\mathrm{C}(3)-\mathrm{C}(4)-\mathrm{N}(5)-\mathrm{O}(2)-\mathrm{N}(4)]$, 平面 1 与平 面 $3[\mathrm{C}(5)-\mathrm{C}(6)-\mathrm{N}(7)-\mathrm{O}(3)-\mathrm{N}(6)$ ] 以及平面 2 与平面 3 之间的二面角分别为 $4.06^{\circ}, 6.63^{\circ}$ 和 $7.8^{\circ}$, 也表明 3 个呋咱 环基本共面. 从二面角 $\mathrm{C}(4)-\mathrm{C}(5)-\mathrm{C}(6)-\mathrm{N}(1)\left(-2.5^{\circ}\right)$, $\mathrm{N}(1)-\mathrm{C}(1)-\mathrm{C}(2)-\mathrm{C}(3)\left(0.9^{\circ}\right), \mathrm{N}(1)-\mathrm{C}(1)-\mathrm{N}(2)-\mathrm{O}(1)$ $\left(179.51^{\circ}\right), \mathrm{N}(6)-\mathrm{C}(5)-\mathrm{C}(6)-\mathrm{N}(1)\left(179.84^{\circ}\right)$ 可以看出 $\mathrm{N}$ 原子和三个呋咱环共面, 即整个环形成大的 $\pi-\pi$ 共轭体 系. 因此该分子的结构比较稳定.

HTFAZ 分子中 $\mathrm{C}(1)-\mathrm{N}(1)(0.1369 \mathrm{~nm}), \mathrm{C}(1)-\mathrm{C}(2)$ $(0.1426 \mathrm{~nm}), \mathrm{C}(2)-\mathrm{C}(3)(0.1458 \mathrm{~nm})$ 的键长与 $\mathrm{C}(6)$ $\mathrm{N}(1)(0.1368 \mathrm{~nm}), \mathrm{C}(4)-\mathrm{C}(5)(0.1455 \mathrm{~nm}), \mathrm{C}(5)-\mathrm{C}(6)$ $(0.1427 \mathrm{~nm})$ 的键长相似, 表明该分子为对称结构.

HTFAZ 分子结构中主要存在的是分子间的氢键(图 2), 仲胺上的 $\mathrm{H}$ 原子与另外一分子 HTFAZ 上的 N 原子 形成氢键. 分子间的氢键如表 6 所示, 该氢键中氢原子 是二配位即 $\mathrm{N}(1)-\mathrm{H}(1) \cdots \mathrm{N}(7)$ 形式存在. 其中 $\mathrm{H} \cdots \mathrm{N}(7)$ 的键长 $(0.219 \mathrm{~nm})$ 为 $\mathrm{N}(1)-\mathrm{H}$ 键长 $(0.084 \mathrm{~nm})$ 的 2.5 倍, 氢原子接近 $\mathrm{N}(1)$, 不是最强氢键 $(\mathrm{X}-\mathrm{H} \cdots \mathrm{Y}, \mathrm{H}$ 原子位于 接近 $\mathrm{X} \cdots \mathrm{Y}$ 中心). 键角 $\mathrm{N}(1)-\mathrm{H}(1) \cdots \mathrm{N}(7)$ 为 $165.7^{\circ}$, 可 能受晶体中原子的排列和堆积引起. 从图 2 可以看出 HTFAZ 分子成层状排列, 层内通过氢键连接, 层间存 在范德华力, 导致分子堆积紧密, 晶体密度达到 1.871 $\mathrm{g} \cdot \mathrm{cm}^{-3}$. 分子间氢键导致分子具有较好的热稳定性. 该 化合物的晶体数据已经收录于剑桥晶体数据库, 编号为 CCDC: 1026193.

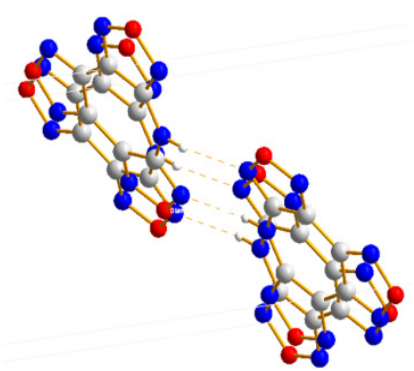

图 2 HTFAZ 的氢键结构

Figure 2 Hydrogen-bonded structure of HTFAZ

\subsection{HTFAZ, ATFAZ 和 BTFZ 的性能计算}

利用 GAUSSIAN 98 程序 ${ }^{[20]}$ 和 VLW 爆轰方程 ${ }^{[21]}$ 计 算的方法, 对目标化合物进行了性能计算, 结果如表 7 . 
表 7 HTFAZ, ATFAZ 和 BTFZ 的部分性能

Table 7 The performances of HTFAZ, ATFAZ and BTFZ

\begin{tabular}{|c|c|c|c|c|c|c|c|c|c|}
\hline Property & Appearance & $\begin{array}{l}\text { Density/ } \\
\left(\mathrm{g} \cdot \mathrm{cm}^{-3}\right)\end{array}$ & Dissolubility & m.p. $/{ }^{\circ} \mathrm{C}$ & $\begin{array}{l}\text { Decomposition } \\
\text { temperature } /{ }^{\circ} \mathrm{C}\end{array}$ & $\begin{array}{c}\text { Detonation } \\
\text { velocity/ } \\
\left(\mathrm{m} \cdot \mathrm{s}^{-1}\right) \\
\end{array}$ & $\begin{array}{l}\text { Detonation pres- } \\
\text { sure/GPa }\end{array}$ & $\begin{array}{l}\text { Enthalpy of } \\
\text { formation/ } \\
\left(\mathrm{kJ} \cdot \mathrm{mol}^{-1}\right)\end{array}$ & $\begin{array}{l}\text { Explosion tem- } \\
\text { perture/K }\end{array}$ \\
\hline HTFAZ & White solid & 1.871 & $\begin{array}{l}\text { Acetone and } \\
\text { DMSO }\end{array}$ & 193.1 & 345.1 & 8416.5 & 32.9 & 733.7 & 5110.6 \\
\hline ATFAZ & White solid & 1.788 & $\begin{array}{l}\text { Acetone and } \\
\text { DMSO }\end{array}$ & $\begin{array}{c}204 \sim \\
207\end{array}$ & 323.7 & 8300 & 30 & 831.2 & 5085.9 \\
\hline BTFZ & White solid & 1.862 & $\begin{array}{l}\text { Acetone and } \\
\text { acetic ether }\end{array}$ & - & 280.2 & 7924.5 & 28.42 & 1752.4 & - \\
\hline $\begin{array}{l}\text { Test } \\
\text { condition }\end{array}$ & Eyeballing & $\begin{array}{l}\text { X-ray single } \\
\text { crystal }\end{array}$ & $\begin{array}{l}\text { Experimental } \\
\text { method }\end{array}$ & DSC & DSC & $\begin{array}{c}\text { VLW } \\
\text { method }\end{array}$ & VLW method & Gaussian 98 & VLW method \\
\hline
\end{tabular}

采用 Gaussian 98 程序 B3LYP16-31 方法计算 ${ }^{[20]}$

从表 7 中可以看出, HTFAZ 与 ATFAZ 相比, 其密

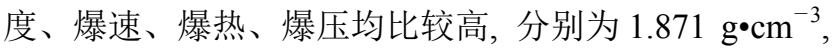
$8416.5 \mathrm{~m} \cdot \mathrm{s}^{-1}, 5110.6 \mathrm{~K}, 32.9 \mathrm{GPa}$, 熔点和生成焓较低, 分别为 $193.1{ }^{\circ} \mathrm{C}, 733.7 \mathrm{~kJ} \cdot \mathrm{mol}^{-1}$. 结果表明, HTFAZ 比 ATFAZ 的能量高, 因为 HTFAZ 的晶体密度较高, 通过 晶体的堆积图可以看出 HTFAZ, 分子共面性好, 层状 分布, 堆积更为紧密, 密度较高. 由于分子间存在氢键 导致其热稳定性更好, 分解点达到 $345.1{ }^{\circ} \mathrm{C}$. 其中 HTFAZ 与 BTFZ 的结构类似, 因此其密度相当, 由于分 子量较大导致其生成焓较高.

\subsection{HTFAZ 与 BTFZ 的热行为}

采用差式扫描量热仪和热重分析仪, $10{ }^{\circ} \mathrm{C} \cdot \mathrm{min}^{-1}$ 的 升温速率条件下获得了 HTFAZ 和 BTFZ 的 DSC 和 TG-DTG 图谱(图 3,4), 考察了在 30 500 ${ }^{\circ} \mathrm{C}$ 条件下的 热稳定性, 结果见图所示. 在 $\mathrm{N}_{2}$ 气氛, 进行 DSC 测试, 流速 $50 \mathrm{~mL} \cdot \mathrm{min}^{-1}$, 温度范围 $30 \sim 500{ }^{\circ} \mathrm{C}$, 在常压曲线 [1]和加压 $1 \mathrm{MPa}$ 曲线[2]测试结果如图 3a 所示.

图 3 可以看出, 曲线[1]在 $193.1{ }^{\circ} \mathrm{C}$, 有一个吸热峰, 为 HTFAZ 的熔化峰. 随着温度继续升高, 在 $252.3{ }^{\circ} \mathrm{C}$ 出现一个缓慢吸热峰, 并没有明显的放热峰. 从加压以 后曲线[2]可以看出, 在 $193.1{ }^{\circ} \mathrm{C}$ 有一个吸热峰, 与常压 下熔点一致; 继续升温, 在 $345.1{ }^{\circ} \mathrm{C}$ 有个明显的放热峰, 在 $295{ }^{\circ} \mathrm{C}$ 以前该化合物汽化没有分解. 两条曲线对比 表明: 该化合物熔化后，在氮气流的吹带下发生了汽化， 然后放热分解, 因此常压 DSC 并未出现放热峰, 而是出 现了缓慢的吸热峰. 从 HTFAZ 的 TG-DTG 曲线(图 3b) 可以看出在程序升温过程 $\left(20 \sim 300{ }^{\circ} \mathrm{C}\right)$ 中, 当温度低于 $116.5{ }^{\circ} \mathrm{C}$ 时失重较少, 累积失重仅为 $3 \%$ 左右, 继续升 温, 最大的失重峰出现在 $241.6{ }^{\circ} \mathrm{C}$, 当温度达到 $270.1{ }^{\circ} \mathrm{C}$ 时质量损失完全，质量损失率达 $98.4 \%$.

从图 4 可以看出, BTFZ 的 DSC 曲线上有个明显的 放热峰 $280.2{ }^{\circ} \mathrm{C}$, BTFZ 没有经历吸热熔化过程, 在固相 状态下直接分解, 迅速放出大量热量; 从图 $4 \mathrm{~b}$ 中 BTFZ
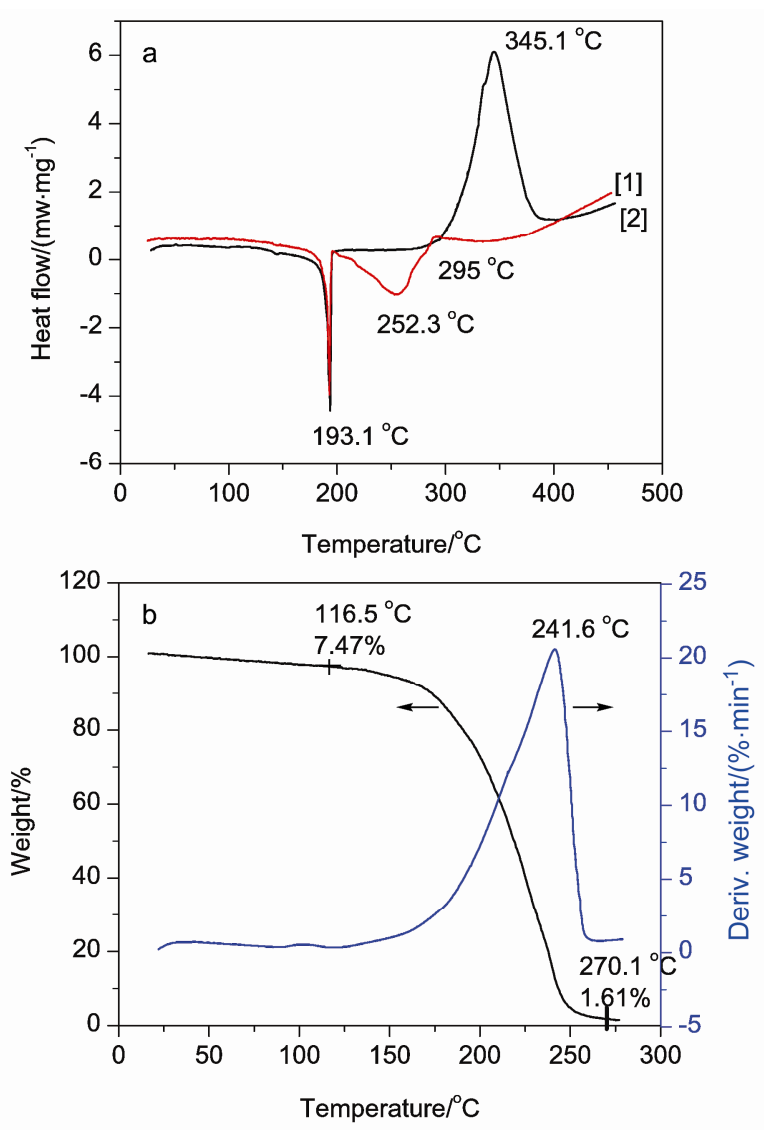

图 3 HTFAZ 的 DSC (a) 和 TG-DTG (b)曲线图

Figure 3 DSC (a) and TG-DTG (b) curve of HTFAZ [1] DSC in atmospheric pressure; [2] DSC in $1 \mathrm{MPa}$

的 TGA 曲线可以看出，当温度低于 $174.8{ }^{\circ} \mathrm{C}$ 时 $\mathrm{BTFZ}$ 的失重较少，累积失重仅为 $3.7 \%$; 在 $174.8{ }^{\circ} \mathrm{C}$ 到 $309.8^{\circ} \mathrm{C}$ 升温过程中快速失重, 失重约为 $83.4 \%$; 到 $365.8{ }^{\circ} \mathrm{C}$ 分解基本完成, 还有部分残渣(约 $12.2 \%$ ), 随着 温度的升高, 残余物质进一步分解.

经过以上分析表明, HTFAZ 在受热过程中先熔化, 在动态氮气吹带下进一步发生熔融态汽化, 在汽化完之 前并没有明显的分解过程; HTFAZ 具有较好的热稳定 性，但高温下易汽化，而化合物 BTFZ 没有熔点，不易 

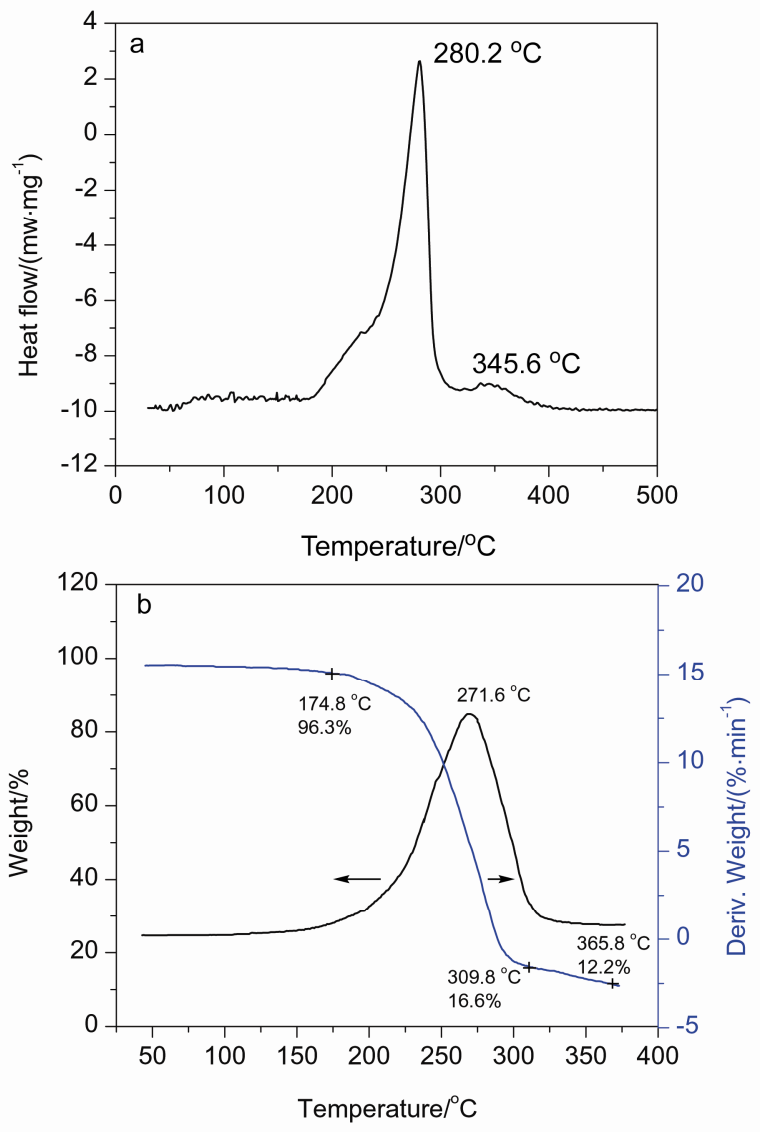

图 4 BTFZ 的 DSC (a) 和 TG-DTG (b)曲线图

Figure 4 DSC (a) and TG-DTG (b) curves of BTFZ

汽化, 直接分解, 分解点为 $280.2{ }^{\circ} \mathrm{C}$.

\section{2 结论}

以 BATF 为原料, 经过氧化、取代、环化反应合成 了两种含能化合物 HTFAZ 与 ATFAZ, 在硝酸-醋酐体 系中氧化 HTFAZ 得到了未见文献报道的新化合物 BTFZ, 采用红外光谱、核磁共振、元素分析和质谱对三 种化合物进行了结构表征; 推测了 HTFAZ 成环反应和 BTFZ 氧化反应机理; 培养了 HTFAZ 单晶, 晶体结构分 析表明: HTFAZ 为单斜晶系, 空间群为 $P 2(1) / n$, 晶体密 度 $1.871 \mathrm{~g} \cdot \mathrm{cm}^{-3}$; 通过计算和实测的方法对 HTFAZ, ATFAZ和 BTFZ 的物化和爆轰性能进行研究, 其密度分 别为 $1.871,1.788,1.862 \mathrm{~g} \cdot \mathrm{cm}^{-3}$, 爆速分别为 8416.5 , $8300,7924.5 \mathrm{~m} \cdot \mathrm{s}^{-1}$. 采用 DSC 和 TG-DTG 研究了 HTFAZ 和 BTFZ 的热分解过程，其分解点分别为 345.1 和 $280.2{ }^{\circ} \mathrm{C}$, 表现出了良好的热稳定性.

\section{3 实验部分}

\section{1 仪器与试剂}

瑞士 BRUKER 公司的 AV500 型(500 MHz)超导核
磁共振仪; 美国热电尼高力公司 NEXUS870 型傅里叶 变换红外光谱仪; 德国 EXEMENTAR 公司 Vario EL-III 型元素分析仪; 日本岛津 GC-MS-QP 2010 Plus 型质谱 仪; 日本岛津公司 LC-2010A 液相色谱仪; 北京泰克仪 器有限公司 X-6 型显微熔点测定仪; 瑞士 BRUKER 公 司 Bruker Smart APEXIICCD 衍射仪; 美国 Nicolet 公司 TA2950 热重仪; 德国 Netzsch 公司 DSC-204HP 型高压 差示扫描量热

浓硫酸、铇酸钠、乙腈、水合肼、双氧水(质量分 数 $50 \%$ )、氨水(质量分数 $25 \% \sim 28 \%$ ) 等均为分析纯; 硝 酸为工业品(质量分数 97\%); 3,4-双(3'-氨基呋咱-4'-基)呋 咱自制 ${ }^{[22]}$.

\section{2 实验方法}

\subsubsection{3,4-双 (3'-硝基呋咱-4'-基)呋咱(BNTF)的合成}

向 $250 \mathrm{~mL}$ 的反应瓶中加入 $50 \mathrm{~mL}$ 的 $50 \%$ 的 $\mathrm{H}_{2} \mathrm{O}_{2}$ 和 $\mathrm{NaWO}_{4} \cdot 2 \mathrm{H}_{2} \mathrm{O}(6.0 \mathrm{~g}, 0.02 \mathrm{~mol})$, 在 $0{ }^{\circ} \mathrm{C}$ 摚拌下, 缓慢 滴加 $60 \mathrm{~mL}$ 的 $98 \%$ 的硫酸, 滴加时间约为 $20 \mathrm{~min}$, 滴加 完毕, 升温至 $30 \sim 35{ }^{\circ} \mathrm{C}$, 分批加入 BATF $(5 \mathrm{~g}, 0.02$ $\mathrm{mol})$, 加料完毕保温 $1 \mathrm{~h}$, 降温至 $10{ }^{\circ} \mathrm{C}$, 倒入 $200 \mathrm{~mL}$ 冰 水中, 过滤、水洗、干燥得白色固体 $5.8 \mathrm{~g}$, 收率 $93 \%$. m.p. $88 \sim 89{ }^{\circ} \mathrm{C} ;{ }^{13} \mathrm{C}$ NMR (DMSO- $d_{6}, 125 \mathrm{MHz}$ ) $\delta$ : 160.17, 142.09, 138.94; IR (KBr) v: 1613 (furzan), 1572, $1384\left(\mathrm{NO}_{2}\right) \mathrm{cm}^{-1}$; MS (EI) m/z: $296\left[\mathrm{M}^{+}\right]$. Anal. calcd for $\mathrm{C}_{6} \mathrm{~N}_{8} \mathrm{O}_{7}$ : C 24.32, N 43.24; found C 24.47, N 43.64.

3.2.27H-三呋咱并 $[3,4-b: 3,4$ '- $f: 3 ", 4 "-d]$ 氮杂环庚三烯 (HTFAZ) 的合成

向 $50 \mathrm{~mL}$ 的反应瓶中加入 $30 \mathrm{~mL}$ 无水乙腈后, 再加 入 $2.0 \mathrm{~g}(6.8 \mathrm{mmol}) \mathrm{BNTF}$, 滴加 $2 \mathrm{~g}(14.2 \mathrm{mmol})$ 的氨水, 在 $25 \sim 30{ }^{\circ} \mathrm{C}$ 搅拌 $2 \mathrm{~h}$. 反应液倒入 $100 \mathrm{~mL}$ 水中, 用 50 $\mathrm{mL}$ 二氯甲烷萃取三次, 分离有机相, 无水硫酸镁干燥 6 $\mathrm{h}$ ，过滤、蒸干得乳白色固体 $0.8 \mathrm{~g}$, 收率 54\%, 纯度 $99 \%$ (HPLC). m.p. $193.1{ }^{\circ} \mathrm{C} ;{ }^{1} \mathrm{H}$ NMR (DMSO- $d_{6}, 500 \mathrm{MHz}$ ) $\delta: 12.42 ;{ }^{13} \mathrm{C}$ NMR (DMSO- $\left.d_{6}, 125 \mathrm{MHz}\right) \delta: 152.44$, 144.47, 137.29; IR (KBr) v: 3200 (NH), 1510, 1480, 1001, 985 (furazan), 1639, $1604(\mathrm{C}=\mathrm{N}) \mathrm{cm}^{-1}$; MS (EI) $\mathrm{m} / z: 219$ $\left[\mathrm{M}^{+}\right]$. Anal. calcd for $\mathrm{C}_{6} \mathrm{HN}_{7} \mathrm{O}_{3}: \mathrm{C} 32.88, \mathrm{H}$ 0.46, $\mathrm{N}$ 44.75; found $\mathrm{C} 32.55, \mathrm{H} 0.38, \mathrm{~N} 44.53$.

$3.2 .37,7^{\prime}-$ 二(呋咱 $\left[3,4-b: 3,4^{\prime}-d: 3^{\prime \prime}, 4^{\prime \prime}-f\right]$ 氮杂环庚三烯) (BTFZ) 的合成

向 $50 \mathrm{~mL}$ 的反应瓶中加入 $10 \mathrm{~mL}$ 工业硝酸, 冷却至 $-10{ }^{\circ} \mathrm{C}$, 分批加入 $1 \mathrm{~g}$ (4.6 mmol) HTFAZ, 在一 10 $0{ }^{\circ} \mathrm{C}$ 缓慢滴加 $\mathrm{Ac}_{2} \mathrm{O}$, 滴加完毕, 室温下反应 $2 \mathrm{~h}$, 反应 液倒入 $100 \mathrm{~mL}$ 冰水中, 过滤、水洗、干燥得白色固体 $1.73 \mathrm{~g}$, 收率 $87 \%$. ${ }^{13} \mathrm{C}$ NMR (DMSO- $\left.d_{6}, 125 \mathrm{MHz}\right) \delta$ : $151.30,143.94,136.59$; IR (KBr) v: 1594, 1496, 1170, 
1003 (furazan) $\mathrm{cm}^{-1}$. Anal. calcd for $\mathrm{C}_{12} \mathrm{~N}_{14} \mathrm{O}_{6}: \mathrm{C} 33.03, \mathrm{~N}$ 44.95; found C 33.15, N 44.82 .

3.2.4 7-氨基三呋咱并 [3,4-b:3,4'-f:3",4"-d] 氮杂环庚 三烯(ATFAZ)的合成

向 $50 \mathrm{~mL}$ 三口烧瓶中加入 BNTF (2.5 g, $8.4 \mathrm{mmol})$ 和 $20 \mathrm{~mL}$ 乙腈, 在 $10 \sim 15{ }^{\circ} \mathrm{C}$ 滴加 $80 \%$ 水合肼 $(1.1 \mathrm{~g}, 17.6$ $\mathrm{mmol}$ )滴加完毕, 升温至 $25 \sim 30{ }^{\circ} \mathrm{C}$ 继续反应 $8 \mathrm{~h}$. 将反 应液倒入 $50 \mathrm{~mL}$ 的水中, 过滤, 用水充分淋洗, 干燥得 乳白色固体 $0.56 \mathrm{~g}$, 收率 $71 \%$, 纯度 $99 \%$. m.p. 204 $206{ }^{\circ} \mathrm{C} ;{ }^{1} \mathrm{H}$ NMR (DMSO- $\left.d_{6}, 500 \mathrm{MHz}\right) \delta$ : 6.157 (s, 2H, $\left.\mathrm{NH}_{2}\right) ;{ }^{13} \mathrm{C}$ NMR (DMSO- $\left.d_{6}, 125 \mathrm{MHz}\right) \delta: 156.63,144.22$, 135.72; IR (KBr) v: 3319, 1632, 1578, 1494, 1423, 990 $\mathrm{cm}^{-1}$. Anal. calcd for $\mathrm{C}_{6} \mathrm{H}_{2} \mathrm{~N}_{8} \mathrm{O}_{3}: \mathrm{C} 30.77, \mathrm{H} 0.85, \mathrm{~N}$ 47.86; found C 30.54, H 0.71, N 47.95.

\subsection{HTFAZ 单晶培养与测定}

\subsubsection{HTFAZ 单晶培养}

将纯度大于 $99.0 \%$ 的 HTFAZ 自制样品, 加入到水 和乙醇(体积比 $1: 1$ )的混合溶液中, 充分溶解后, 过滤 出不溶物得到无色溶液, 置于干净的培养瓶中, 室温下 放置一段时间后，得到无色透明晶体.

\subsubsection{HTFAZ 单晶测定}

选取尺寸为 $0.38 \mathrm{~mm} \times 0.31 \mathrm{~mm} \times 0.26 \mathrm{~mm}$ 的 HTFAZ 单晶, 室温下置于 Smart apex II CCD 衍射仪上, 经过石墨单色器单色化的 Mo K $\alpha$ 射线 $(\lambda=0.071073 \mathrm{~nm})$, 以 $\omega / 2 \theta$ 扫描方式, 在 $2.64^{\circ} \leqslant \theta \leqslant 28.27^{\circ},-9 \leqslant h \leqslant 8$, $-8 \leqslant k \leqslant 8,-20 \leqslant l \leqslant 21$ 范围内共收集到 4607 个衍射 点, 其中独立衍射点 1875 个 $\left(R_{\mathrm{int}}=0.0199\right), 1754$ 个 $[I>$ $2 \sigma(I)]$ 衍射点用于结构测定和修正. 晶体结构由程序 SHELXS-97 和 SHELXL-97 直接法解出 ${ }^{[23,24]}$, 经多轮 Fourier 合成获得全部非氢原子. 全部非氢原子的坐标 及各向异性热参数, 采用 $w=1 /\left[\sigma^{2}\left(F_{\mathrm{o}}{ }^{2}\right)+(0.0486 P)^{2}+\right.$ $\left.0.1288 P], P=\left(F_{\mathrm{o}}{ }^{2}+2 F_{\mathrm{c}}{ }^{2}\right) / 3\right)$, 经全矩阵最小二乘法修正 及收敛。

辅助材料(Supporting Information) 目标化合物 7-氨 基-三呋咱并 $\left[3,4-b: 3^{\prime}, 4^{\prime}-f: 3^{\prime \prime}, 4^{\prime \prime}-d\right]$ 氮杂环庚三烯(ATFAZ) 和 7,7'- 二(呋咱 $\left[3,4-b: 3,4^{\prime}-d: 3^{\prime \prime}, 4^{\prime \prime}-f\right]$ 氮杂环庚三 烯)(BTFZ)的结构鉴定图谱. 这些材料可以免费从本刊 网站(http://sioc-journal.cn/)上下载.

\section{References}

[1] Zhang, J. H.; Shreeve, J. M. J. Am. Chem. Soc. 2014, 136, 4437.

[2] Yin, P.; Zhang, Q. H.; Zhang, J. H.; Parrish, D. A.; Shreeve, J. M. J. Mater. Chem. A 2013, 1, 7500.

[3] Fischer, N.; Klapötke, T. M.; Reymann M.; Stierstorfer, J. Eur. J. Inorg. Chem. 2013, 8, 2167.
[4] Zhou, Y.-S.; Zhou, C.; Wang, B.-Z.; Li, J.-K.; Huo, H.; Zhang, Y.-G.; Wang, X.-J.; Luo, Y.-F. Chin. J. Org. Chem. 2011, 19, 509 (in Chinese).

(周彦水, 周诚, 王伯周, 李健康, 霍欢, 张叶高, 王锡杰, 罗义 芬, 有机化学, 2011, 19, 509.)

[5] Li, H.; Zhao, F.-Q.; Gao, H.-X.; Tong, J.-F.; Wang, B.-Z.; Zhai, L.-J.; Huo, H. Inorg. Chim. Acta 2014, 432, 256.

[6] Klapötke, T. M.; Mayr, N.; Stierstorfer, J.; Weyrauther, M. Chem. Eur. J. 2014, 20, 1410.

[7] Li, X.-Z.; Wang, B.-Z.; Li, H.; Li, Y.-N.; Bi, F.-Q.; Huo, H.; Fan, X.-Z. Chin. J. Org. Chem. 2012, 32, 1975 (in Chinese). (李祥志, 王伯周, 李辉, 李亚南, 毕福强, 霍欢, 樊学忠, 有机 化学, 2012, 32, 1975.)

[8] Hu, H.-X.; Zhang, Z.-Z.; Zhao, F.-Q. Acta Armamentarii 2004, 25, 155 (in Chinese).

(胡焕性，张志忠，赵凤起，兵工学报, 2004, 25, 155.)

[9] Wang, B.-Z.; Jia, S.-Y.; Wang, X.-J. CN 201010047638, 2010.

[10] Wang, X.-J.; Wang, B.-Z.; Jia, S.-Y. Chin. J. Energy Mater. 2012, 20, 258 (in Chinese) (王锡杰，王伯周，贾思媛，含能材料, 2012, 20, 258.)

[11] Zhou, Y.-S.; Wang, B.-Z.; Wang X.-J. Chin. J. Energy Mater. 2012, 20, 137 (in Chinese). (周彦水, 王伯周, 王锡杰, 含能材料, 2012, 20, 137.)

[12] Zhou Y.-S.; Wang B.-Z.; Wang X.-J. Chin. J. Synth. Chem. 2012, 20, 147 (in Chinese).

(周彦水, 王伯周, 王锡杰, 合成化学, 2012, 20, 147.)

[13] Astrat'ev A. A.; Stepanov A. I.; Dashko D. V. 16th International Seminar New Trends in Research of Energetic Materials (NTREM), Pardubice, Czech Republic, April 10 12, 2013, 474.

[14] Stepanov, A. I.; Dashko, D. V; Astrat'ev, A. A. 15th International Seminar New Trends in Research of Energetic Materials (NTREM), Pardubice, Czech Republic, April 18 20, 2012, 301.

[15] Zhou, Y.-S.; Zhang, Z.-Z.; Li, J.-K. Chin. J. Explos. Propellants 2005, 28, 43 (in Chinese).

(周彦水, 张志忠, 李健康, 火炸药学报, 2005, 28, 43.)

[16] Zhou, Y.-S.; Wang, B.-Z.; Li, J.-K.; Zhou, C.; Hu, L.; Chen, Z.-Q.; Zhang, Z.-Z. Acta Chim. Sinica 2011, 69, 1673 (in Chinese). (周彦水, 王伯周, 李建康, 周诚, 胡岗, 陈智群, 张志忠, 化学 学报, 2011, 69, 1673.)

[17] Xing, Q.-Y. Organic Chemistry, Advanced Education Press, Beijing, 1980, p. 932. (邢其毅, 有机化学, 高等教育出版社, 北京, 1980, p. 932.)

[18] Zhai, L.-J.; Wang, B.-Z.; Huo, H.-H.; Li, H.; Li, Y.-N.; Huang, X.-P.; Liu, N.; Fang, X.-Z. Chin. J. Org. Chem. 2013, 33, 1755 (in Chinese).

(翟连杰, 王伯周, 霍欢, 李辉, 李亚南, 黄新萍, 刘宁, 樊学忠, 有机化学, 2013, 33, 1755.)

[19] Zhang, Q. H.; Zhang, J. H.; Parrish, D. A.; Shreeve, J. M. Chem. Eur. J. 2013, 19, 11000.

[20] Frisch, M. J.; Trucks, G. W.; Schlegel, H. B. Gaussian 98, Gaussian, Inc. of Walling, PA, 2009

[21] Wu, X.; Long, X.-P.; He, B. Sci. China, B 2008, 38, 1129 (in Chinese).

(吴雄, 龙新平, 何碧，中国科学(B 辑), 2008, 38, 1129.)

[22] Jia, S.-Y.; Zhang, H.-H.; Wang, B.-Z. Chin. J. Energy Mater. 2013, 21, 289 (in Chinese).

(贾思媛, 张海昊, 王伯周, 含能材料, 2013, 21, 289.)

[23] Sheldrick, G. M. SHELXL-97, Program for Crystal Structure Refinement, University of Göttingen, Germany, 1997.

[24] Sheldrick, G. M. SHELXTS-97, Program for Crystal Structure Solution, University of Göttingen, Germany, 1997.

(Zhao, C.) 